Revue

Revue de l'histoire des religions

del'histoire des religions

Thierry FAVIER, Le Chant des muses chrétiennes.

Cantiques spirituels et dévotion en France (1685-1715)

Paris, Société française de musicologie, 2008, 23,5 cm, 404 p.

(«Publications de la Société française de musicologie », $3^{\mathrm{e}}$ série, t. $\mathrm{X}$ ), $38 €$.

Fabien Guilloux

\title{
OpenEdition
}

Journals

Édition électronique

URL : http://journals.openedition.org/rhr/7752

DOI : 10.4000/rhr.7752

ISSN : $2105-2573$

Éditeur

Armand Colin

Édition imprimée

Date de publication : 1 mars 2011

Pagination : 136-139

ISBN : 978-2200-92685-4

ISSN : 0035-1423

Référence électronique

Fabien Guilloux, "Thierry favier, Le Chant des muses chrétiennes. Cantiques spirituels et dévotion en

France (1685-1715) », Revue de l'histoire des religions [En ligne], 1 | 2011, mis en ligne le 30 mai 2011, consulté le 11 octobre 2020. URL : http://journals.openedition.org/rhr/7752 ; DOI : https://doi.org/

10.4000/rhr.7752

Ce document a été généré automatiquement le 11 octobre 2020

Tous droits réservés 


\section{Thierry FAVIER, Le Chant des muses chrétiennes. Cantiques spirituels et dévotion en France (1685-1715)}

Paris, Société française de musicologie, 2008, 23,5 cm, 404 p. («Publications de la Société française de musicologie », $3^{\mathrm{e}}$ série, t. X), $38 €$.

Fabien Guilloux

\section{RÉFÉRENCE}

Thierry FAVIER, Le Chant des muses chrétiennes. Cantiques spirituels et dévotion en France (1685-1715), Paris, Société française de musicologie, 2008, 23,5 cm, 404 p. (« Publications de la Société française de musicologie ", $3^{e}$ série, t. X), $38 €$.

1 Fruit d'une thèse de doctorat soutenue à la Sorbonne en 1996, l'ouvrage de Thierry Favier étudie un aspect méconnu du chant religieux français : les «productions de la poésie lyrique sacrée, mises en musique sous forme savante entre 1685 et 1715 » (p. 7). Le livre se compose de trois grandes sections comprenant successivement l'étude proprement dite, une série d'annexes documentaires dont un riche et détaillé Catalogue des cantiques spirituels savants (1672-1715) et s'achève par la bibliographie, les index et tables.

2 Comme le rappelle l'auteur, l'apparition du cantique spirituel savant est étroitement liée à l'émergence d'un corpus de poésies originales qui, sur les modèles de Jean Racine et Jean Crasset, se diffusent sous la forme de recueils d'auteurs ou d'anthologies et se démarquent du genre encore largement apprécié de la paraphrase biblique. Le second chapitre focalise ensuite l'attention sur la figure et l'œuvre du compositeur et théoricien Bénigne de Bacilly (ca. 1625-1690) puis ses épigones (Nicolas Fleury, Toinon, Des Forêts) qui dans les années 1670-1690 militèrent en faveur d'une réforme de l'air spirituel, réforme dont l'influence semble toutefois s'être limitée à quelques cénacles 
parisiens et versaillais. L'évolution stylistique du cantique spirituel strophique traditionnel vers sa forme savante à sections musicales différenciées fait l'objet du troisième chapitre. S'appuyant sur un corpus restreint (une dizaine d'œuvres), l'auteur distingue ici deux tendances: une première, représentée par Jean-Baptiste Moreau, Michel-Richard de Lalande, un certain Duhalle et Pascal Colasse, qui « tend à maintenir, malgré la différenciation des strophes, une unité dans l'expression et le sentiment " (p. 82) ; une seconde qui, développée par Michel Farinel, Pierre-César Abeille, Jean-Noël Marchand, Antonia Bembo, Claude Oudot et Jacques Morel, « nettement influencée par l'évolution de la musique dramatique, favorise au contraire les expressions contrastées et la dramatisation du discours " (ibidem). Malheureusement, faute de documentation fiable, il est aujourd'hui difficile d'établir avec certitude «la fréquence, le lieu et les circonstances [...] ou la nature du public concerné » (p. 129) par ce répertoire. Dans le quatrième chapitre, l'auteur se trouve ainsi réduit à énumérer des lieux et facteurs culturels connus de l'historiographie musicale : la cour de Louis XIV et le rôle joué par des auteurs comme Élisabeth-Sophie Chéron, Jacques Testu et Jean Racine, les éditeurs parisiens, les communautés religieuses et les maisons d'éducation, dont celle de SaintCyr. L'étude s'achève par un chapitre consacré aux enjeux éthiques et esthétiques de ce répertoire replacé dans le cadre général de la Réforme catholique. L'auteur y décèle un glissement « de la pédagogie au plaisir » (p.193) manifesté par le rejet de la parodie qu'il analyse à partir de la satire La Poésie et la Musique (1695) de Pierre Bellocq et dont il relie les propos aux prises de positions jansénistes concernant la musique.

3 Si l'apport documentaire est indéniablement le point fort de l'ouvrage de T. Favier, on émettra quelques réserves quant à ses analyses. En premier lieu, il est regrettable que la chronologie du champ d'étude annoncée en titre (1685-1715) ne soit ni exposée ni justifiée. On a d'autant plus de difficultés à comprendre la logique suivie par l'auteur que le deuxième chapitre repose essentiellement sur des publications antérieures à 1685 et que le Catalogue annonce couvrir une période allant de 1672 à 1715, période qu'il dépasse d'ailleurs en intégrant les rééditions postérieures à 1715 (p. 314, 318). Ce choix de la courte durée empêche surtout de situer les œuvres étudiées dans le cadre plus vaste de la production musicale spirituelle des $\mathrm{XVII}^{\mathrm{e}}$ et $\mathrm{XVIII}^{\mathrm{e}}$ siècles. À la suite de D. Launay (La Musique religieuse en France du concile de Trente à 1804, Paris, 1993), il est ici bon de rappeler - l'auteur y fait brièvement allusion (p. 6-7) - que les techniques de la paraphrase poétique et du contrafactum musical ont toujours été des modalités à la fois concurrentes et complémentaires aux compositions originales (textes et musiques) en France depuis la fin du $\mathrm{XVI}^{\mathrm{e}}$ siècle. Bien qu'exceptionnelle dans sa réalisation, la réforme de Bacilly s'inscrit donc dans cette tradition et gagnerait à être confrontée à celle antérieure proposée par Irénée d'Eu et Denis Macé qui, dans les Cantiques spirituels (Paris, 1639), faisaient déjà des choix conscients et programmatiques en ce sens (p. 193-4); ceci expliquerait d'ailleurs la réédition de l'ouvrage en 1699, 1717 et 1728 (p. 147, 149). Le même rapprochement pourrait être fait avec les trois Livre d'Airs de dévotion publiés par François Berthod entre 1656 et 1662 . T. Favier les considère comme les archétypes d'un genre (le contrafactum) contre lequel s'érigerait la réforme de Bacilly (p. 35, 154, 190). C'est toutefois oublier que le troisième Livre d'Airs de dévotion (Paris, 1662) comporte déjà une série de cantiques inédits mis en musique par Thomas Gobert ce qui, là encore, expliquerait le succès éditorial de ce recueil réimprimé en 1699, 1717 et 1728 (p. 147, 149) - non mentionnée, la réédition de 1728 est signalée par L. Guillo (Pierre I Ballard et Robert III Ballard. Imprimeurs du roy pour la musique (1599-1673), Liège, 2003, cat. $\mathrm{n}^{\circ}$ 1728-A). Sur le même plan, il conviendrait aussi de nuancer 
l'affirmation selon laquelle «la condamnation du répertoire profane, rapidement évoquée [dans les préfaces de B. de Bacilly], est beaucoup moins agressive que dans bon nombre de recueils des décennies précédentes, les propos les plus virulents venant de certains ecclésiastiques comme le Père Irénée d'Eu » (p. 190). Le renvoi sans précision à $\mathrm{Du}$ vray chemin du Ciel (Paris, 1639) ne paraît pas ici pertinent vu que la seule « virulence » décelable du religieux réside dans ces conseils plus moraux qu'esthétiques adressés au méditant: "Ie dis neantmoins autant respectueusement qu'il m'est possible, que l'ame doit beaucoup prendre garde que ces airs mondains ne luy fassent subtilement couler dans le cœur par l'aureille des sentimens mondains" (Du vray chemin du Ciel, p. 211-2). Il aurait été ici plus judicieux de citer certains textes du capucin Yves de Paris auquel il est brièvement fait allusion (p. 189), dont le ton critique sur le plan pastoral trouve des échos dans le Manuel de la mission (Troyes, 1702) d'Albert de Paris (p. 194).

Pour T. Favier, la figure et l'œuvre de B. de Bacilly constituent à la fois le point de départ et le modèle esthétique abouti du cantique spirituel à l'aune desquels est évalué l'ensemble du répertoire. Il y a cependant un risque à ce postulat : celle de surévaluer une forme et une réforme musicale tout compte fait marginales et originales dans l'histoire du chant spirituel. Le fait que les ouvrages de Bacilly et la majorité du corpus inscrit au Catalogue soient imprimés chez des concurrents de la maison Ballard ou se soient diffusés par le manuscrit sont des indicateurs peut-être insuffisamment pris en compte par l'auteur qui trahissent l'influence probable de quelques cénacles érudits et dévots proches des milieux jansénistes et non dénués d'esprit de coterie ou de partisannerie. D'autre part, l'approche esthétique basée sur ce postulat tend à déprécier la production postérieure à cette réforme qui ne serait du coup que «désintégration stylistique du cantique spirituel» (p.67-79). Ce positionnement soulève une question de fond. Lorsqu'à propos du recueil d'Airs spirituels des meilleurs autheurs (Paris, C. Ballard, 1701) l'auteur écrit que «l'insertion de chansons spirituelles d'une qualité médiocre affaiblit [...] la portée artistique de cette anthologie sur le plan esthétique autant que spirituel et témoigne des préoccupations commerciales de l'éditeur, soucieux de toucher le public le plus large possible» (p. 64), doit-on conclure que l'enjeu esthétique est l'unique raison d'être de ce répertoire? Sans nier cet aspect et celui économique il convient de rappeler que l'objet d'étude est avant tout « musique d'usage " inhérente à une pastorale et plus encore à un art de la méditation que T. Favier ne semble pas entrevoir (cf. C. Gordon-Seifert, « From Impurity to Piety: Mid 17th-Century French Devotional Airs and the Spiritual Conversion of Women », The Journal of Musicology, 22/2 (2005), p. 268-291). Concernant le contexte de production et de consommation de ce répertoire, l'auteur aurait ainsi eu intérêt à s'appuyer sur certains travaux réalisés sur les milieux réguliers italiens et espagnols (R. Kendrick, Celestial Sirens. Nuns and their Music in Early Modern Milan, Oxford, 1996; K. Montford, Music in the Convents of Counter-Reformation Rome, Ph.D., Rutgers University, 1999; C. R. Baade, Music and Music-making in Female Monasteries in Seventeenth-century Castille, Ph.D., Duke University, 2001), qui proposent des points de comparaisons tant factuels que méthodologiques susceptibles de décloisonner une réflexion et une démarche somme toute largement tributaire des travaux d'A. Gastoué (Le Cantique populaire en France, Lyon, 1924) et de D. Launay (op. cit.).

5 Si l'on est redevable à T. Favier d'avoir donné en annexe la transcription de la satire de P. Bellocq (p. 243-247), on regrettera l'absence des préfaces de Bacilly. Concernant la bibliographie, outre les réserves déjà signalées par J.-P. Montagnier (Revue de 
musicologie, 95/1 (2009), p. 236-237), on ne peut que déplorer l'absence quasi-totale de références à des travaux étrangers, certes peu nombreux et concernant indirectement le sujet, mais qui s'avéreraient de précieux alliés pour pallier les limites précédemment évoquées; on pense notamment aux études classiques de D. Packer («Collections of Chaste Chansons for the Devout Home (1613-1633)», Acta Musicologica, LXI/2 (1989), p. 175-216) et G. Stefani (Musica barocca. Poetica e ideologia, Milano, 1974). Signalons enfin que La Despouille d'Aegypte (Paris : Ballard, 1629) de Vincent de Rouen figure sans nom d'auteur dans la bibliographie (p. 348).

\section{AUTEURS}

\section{FABIEN GUILLOUX}

Centre d'Études Supérieures de la Renaissance, Tours. 